\title{
Study of cosmogenic activation in copper for rare event search experiments
}

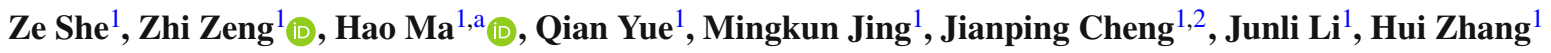 \\ ${ }^{1}$ Key Laboratory of Particle and Radiation Imaging (Ministry of Education) and Department of Engineering Physics, Tsinghua University, \\ Beijing 100084, China \\ ${ }^{2}$ College of Nuclear Science and Technology, Beijing Normal University, Beijing 100875, China
}

Received: 15 June 2021 / Accepted: 13 November 2021 / Published online: 27 November 2021

(C) The Author(s) 2021, corrected publication 2021

\begin{abstract}
Rare event search experiments using germanium detectors are performed in underground laboratories to minimize the background induced by cosmic rays. However, the cosmogenic activation of cupreous detector components on the ground generates long half-life radioisotopes and contributes to the background level. We measured cosmogenic activation with $142.50 \mathrm{~kg}$ of copper bricks after 504 days of exposure at an altitude of $2469.4 \mathrm{~m}$ outside the China Jinping Underground Laboratory (CJPL). The specific activities of the cosmogenic nuclides produced in the copper bricks were measured using a low-background germanium gamma-ray spectrometer at CJPL. The production rates at sea level, in units of nuclei $/ \mathrm{kg} /$ day, were $18.6 \pm 2.0$ for ${ }^{54} \mathrm{Mn}, 9.9 \pm 1.3$ for ${ }^{56} \mathrm{Co}, 48.3 \pm 5.5$ for ${ }^{57} \mathrm{Co}, 51.8 \pm 2.5$ for ${ }^{58} \mathrm{Co}$, and $39.7 \pm 5.7$ for ${ }^{60} \mathrm{Co}$. The measurement will help to constrain cosmogenic background estimation for rare event searches using copper as a detector structure and shielding material. Based on the measured production rates, the impact of the cosmogenic background in cupreous components of germanium detectors on the next generation CDEX-100 experiment was assessed with the expected exposure history above ground.
\end{abstract}

\section{Introduction}

Rare event search experiments, such as dark matter direct detection and neutrinoless double beta decay experiments, are conducted in deep underground laboratories with passive and active shields, and built with materials that are especially radiopure, to effectively reduce their intrinsic background [1-6]. Under ultra-low background conditions, the background induced in the detector's materials on the sur- face could become even more prominent than that from the residual contamination of the primordial nuclides.

With high radiopurity and attractive mechanical properties, copper is widely used in the innermost shields or parts of detectors in rare event search experiments $[3,4,7-10]$. However, radioisotopes produced by cosmogenic activation during manufacturing, transport, and storage can increase the background level of copper. Among these cosmogenic nuclides, short-lived nuclides like ${ }^{59} \mathrm{Fe}$ and ${ }^{48} \mathrm{~V}$ decay rapidly when stored in underground laboratories, while long-lived nuclides like ${ }^{60} \mathrm{Co}$ and ${ }^{54} \mathrm{Mn}$ remain and continue to contribute to the detector's background level [11,12].

The China dark matter experiment (CDEX), aiming at direct dark matter detection and the study of neutrinoless double beta decay of ${ }^{76} \mathrm{Ge}$, operates p-type point-contact germanium detectors (PPCGe) at the China Jinping Underground Laboratory (CJPL) [7,13-16]. The copper used in the PPCGe detectors of the CDEX experiment leads to a crucial background contribution, which requires assessment due to cosmogenic activation in copper to establish the background model for the CDEX experiment. Although the production rates of cosmogenic activation in copper have been investigated via simulations and measurements, discrepancies exist among the results [17-21].

In this study, we present the measurements of the cosmogenic activation in copper samples after exposure to cosmic rays. Corresponding radionuclide production rates are also calculated by Monte Carlo simulations and compared with the measured results. Finally, we simulate the background spectra from cosmogenic radionuclides in the copper components of the PPCGe detector array used in the future CDEX experiment under the expected exposure history of detectors above the ground.

\footnotetext{
a e-mail: mahao@mail.tsinghua.edu.cn (corresponding author)
} 

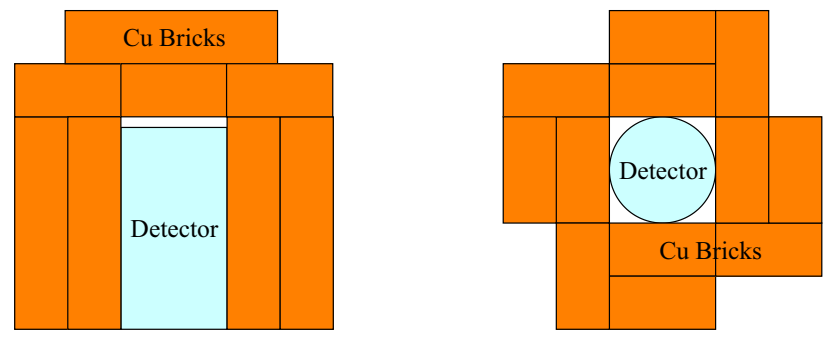

Fig. 1 Schematic diagram of placement of OFHC copper bricks in spectrometer: right view (left) and top view (right)

\section{Experiment and methods}

Sixteen oxygen-free high thermal conductivity (OFHC) copper bricks (their chemical purities are greater than $99.995 \%$ with natural isotope abundance) with a total mass of $142.50 \mathrm{~kg}, 20 \times 10 \times 5 \mathrm{~cm}$ in size, were exposed to cosmic rays and measured in this study. The cosmic-ray muon flux in the CJPL is suppressed by eight orders of magnitude compared to surface laboratories due to $2400 \mathrm{~m}$ rock overburden $[22,23]$. These OFHC copper bricks have been housed in the CJPL for more than four years. The OFHC copper samples were measured to evaluate the residual cosmogenic radioactivity before activation on the surface with a lowbackground germanium gamma-ray spectrometer GeTHU at CJPL, with a background rate between 100 and $2700 \mathrm{keV}$ of $0.510 \pm 0.006 \mathrm{cts} / \mathrm{kg} / \mathrm{min}$ [24]. To enhance the detection efficiency, the germanium detector of GeTHU was surrounded by these copper sample bricks, as shown in Fig. 1. The background spectrum was measured from 12 Aug 2019, to 28 Aug 2019, as depicted in Fig. 2; the characteristic gammaray peaks from cosmogenic radionuclides were not statistically significant and were lower than the minimum detectable activities (MDA) of GeTHU.

These copper bricks were exposed to cosmic rays for 504 days, from Aug. 31, 2019, to Jan. 16, 2021, at an altitude of $2469.4 \mathrm{~m}$ in the vicinity of CJPL. The specific activities of cosmogenic radionuclides inside these copper bricks were calculated by spectral analysis, where the net area of a peak was determined under the assumption of a linear background [25]. The detection efficiencies for concerned radionuclides were simulated by Geant4.10.06 [26-28]. The specific activities of cosmogenic radionuclides can be deduced by combining the production and decay rates,

$A=R\left(1-e^{-\lambda t}\right)+A_{0} e^{-\lambda t}$,

where $A$ is the specific activity, and $R$ denotes the production rate of a particular cosmogenic radionuclide; $\lambda$ is the decay constant and $t$ is the exposure time; $A_{0}$ is the specific activity before exposure. The specific activities of the cosmogenic radionuclides were unsaturated after 504 days of exposure for those with long half-lives, such as ${ }^{60} \mathrm{Co}$. We applied the exponential hypothesis shown in Eq. (1) with zero $A_{0}$ to calculate the production rates, as cosmogenic isotopes were not quantified in the pre-exposure measurement of the copper samples. The decay of cosmogenic radionuclides during the measurements was also considered, especially for those with short half-lives, such as ${ }^{58} \mathrm{Co}$.

The cosmogenic production rate $R$ can be calculated and simplified as

$R=\int \frac{d \Phi(E)}{d E} \sigma(E) d E \approx \Phi_{t o t} \int \frac{d \phi(E)}{d E} \sigma(E) d E \propto \Phi_{t o t}$,

where $\frac{d \Phi(E)}{d E}$ is the energy spectrum of cosmic rays, $\frac{d \phi(E)}{d E}$ is the energy spectrum normalized to an incident particle; $\sigma(E)$ is the cross section of cosmogenic activation for cosmic rays, and $\Phi_{t o t}$ is the flux of cosmic rays. As the energy spectra of a neutron differs slightly by altitude within $20 \mathrm{~km}$ [29], its normalized energy spectra at different altitudes can be considered as a constant. Therefore, the cosmogenic production rates are directly proportional to the intensity of the cosmicray flux, which, at a given altitude, can be parametrized by an exponential [30],

$\Phi_{t o t, H}=\Phi_{t o t, 0} e^{\frac{p(H)-p(0)}{L}}$,

where $\Phi_{t o t, H}$ and $\Phi_{t o t, 0}$ indicate the flux of certain particles in the cosmic ray at altitudes $H$ and sea level, respectively; $p(H)$ is the atmospheric pressure at altitude $H[30,31]$, and $L$ is the typical absorption length for a certain cosmic-ray component. It is generally considered that the production rates of cosmogenic activations are dominated by neutrons, so the absorption length of the neutron is a suitable choice $[21,32]$. Cosmogenic production rates are proportional to the neutron flux, and the same exponential correction can be applied to obtain the corresponding production rates at sea level,

$R(H)=R(0) e^{\frac{p(H)-p(0)}{L}}$.

For comparison, the cosmogenic production rates were also simulated by Geant 4 with the physical list QGSP_INCLXX [27,28,33]. CRY - 1.7 [34] provides the energy spectra of different cosmic-ray components (neutron, proton, muon, gamma, electron, and pion) at sea level for the Geant 4 simulation. In this simulation, the cosmic particles, including the neutron, proton, muon, and gamma from CRY - 1.7 were used by the event generator. We could count each product after interactions with the copper sample to calculate the production rate. 


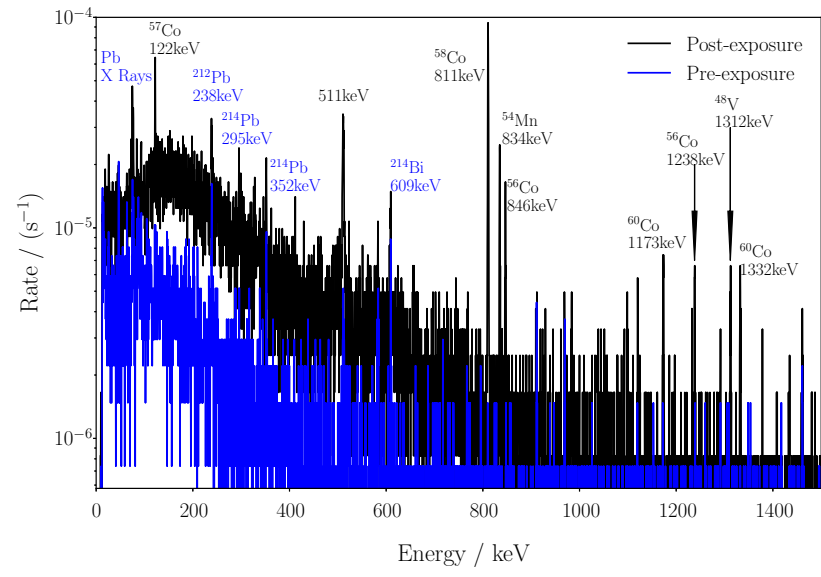

Fig. 2 Measured spectra of OFHC copper samples pre- (blue) and post-exposure (black) with a bin-size of $0.36 \mathrm{keV}$. Gamma-ray peaks induced by cosmogenic activations and primordal radionuclides are also indicated

\section{Cosmogenic activation in copper}

The copper bricks were measured during Jan. 16-30, 2021. The spectrum after exposure is also shown in Fig. 2, where the selected gamma-ray peaks of cosmogenic radionuclides are marked. In addition to the characteristic gamma-ray peaks from cosmogenic radionuclides, there also exist several gamma-ray peaks related to the primordial radionuclides and the $511 \mathrm{keV}$ annihilation peak. Compared with the preexposure spectrum, the background level worsens due to the breakdown of the low-radon air system of the laboratory when measuring these bricks after exposure. GeTHU has a radon mitigation system flushing its sample space with the low-radon air (with boil-off nitrogen gas previously) [24]. Without flushing, the concentrations of radon and its progenies in the sample space increase, and the background rate in the energy region from 238 to $609 \mathrm{keV}$ is higher, as shown in Fig. 2. However, these cosmogenic gamma-ray peaks are still significant enough to warrant calculation of their specific activities. Following gamma-ray spectral analysis, the specific activities of these cosmogenic radionuclides were calculated, and are listed in Table 1.

Table 1 Measured specific activities of cosmogenic radionuclides (with $1 \sigma$ uncertainty) in copper samples corresponding to the values on January 16, 2021. Gamma-ray peaks with better statistics were
Table 2 compares the cosmogenic production rates (obtained from Eq. (1) with $\mathrm{A}_{0}=0$ and Eq. (4)), after altitude correction, with results from previous studies. The results of our measurements are generally consistent with those of our simulation with Geant4 (as shown in Table 2) except for ${ }^{56} \mathrm{Co}$. The measured production rate of ${ }^{57} \mathrm{Co}$ was about $3 \%$ less than simulated, while that of ${ }^{56} \mathrm{Co}$ was $49 \%$ of the simulated result. Our measurements are relatively close to those of Ref. [19]. The discrepancies of production rates with the literature could be due to different cross-section databases, uncertainties of altitude correction, and the fluxes and energy spectra of cosmic rays at different experimental latitudes. Due to its short half-life, 77.2 days, ${ }^{56} \mathrm{Co}$ will decay rapidly when stored underground, resulting in a negligible background contribution. Thus, the difference between simulation and measurement is not a prominent obstacle to the assessment of the cosmogenic background of the underground experiments. Previous Geant4 simulation results [20] differ from this work mainly due to the differences of the physics lists "shielding" and "QGSP_INCLXX" in these two simulations.

\section{Background assessment in PPCGe detector}

The next phase of the CDEX experiment, CDEX-100, will operate about $100 \mathrm{~kg}$ of germanium detectors immersed in liquid nitrogen in a $1725-\mathrm{m}^{3}$ cryostat at Hall $\mathrm{C}$ of the second phase of CJPL. The specific activities of cosmogenic radionuclides are calculated following their production rates and expected exposure history. The total exposure period is a combination of manufacturing and transportation above ground and storage underground. Their specific activities can be calculated as

$$
\begin{aligned}
A= & R(0) e^{\left(p\left(H_{1}\right)-p(0)\right) / L}\left(1-e^{-\lambda t_{\text {manu }}}\right) e^{-\lambda\left(t_{\text {trans }}+t_{\text {cool }}\right)} \\
& +F \times R(0) e^{\left(p\left(H_{2}\right)-p(0)\right) / L}\left(1-e^{-\lambda t_{\text {trans }}}\right) e^{-\lambda t_{\text {cool }}},
\end{aligned}
$$

where $R(0)$ is the measured production rate at sea level (Table 2); $H_{1}$ and $H_{2}$ are the altitudes of the factory and transportation above ground, respectively; $t_{\text {manu }}, t_{\text {trans }}$ and

selected to determine specific activities when radionuclides had two or more gamma-ray peaks. Only statistical uncertainties are considered

\begin{tabular}{lrcl}
\hline Radionuclide & Half-life (day) & Peak energy $(\mathrm{keV})$ & Specific activity $(\mathrm{mBq} / \mathrm{kg})$ \\
\hline${ }^{54} \mathrm{Mn}$ & 312.20 & 834.8 & $0.87 \pm 0.10$ \\
${ }^{56} \mathrm{Co}$ & 77.24 & 846.8 & $0.65 \pm 0.08$ \\
${ }^{57} \mathrm{Co}$ & 271.74 & 122.1 & $2.42 \pm 0.28$ \\
${ }^{58} \mathrm{Co}$ & 70.86 & 810.8 & $3.39 \pm 0.17$ \\
${ }^{60} \mathrm{Co}$ & 1925.28 & 1173.2 & $0.46 \pm 0.07$ \\
\hline
\end{tabular}


Table 2 Production rates of cosmogenic radionuclides in copper at sea level (unit: nuclei $\mathrm{kg}^{-1} \mathrm{day}^{-1}$ ). Only statistical uncertainties are considered

\begin{tabular}{|c|c|c|c|c|c|c|}
\hline & \multirow[t]{2}{*}{ Method } & \multicolumn{5}{|l|}{ Nuclides } \\
\hline & & ${ }^{54} \mathrm{Mn}$ & ${ }^{56} \mathrm{Co}$ & ${ }^{57} \mathrm{Co}$ & ${ }^{58} \mathrm{Co}$ & ${ }^{60} \mathrm{Co}$ \\
\hline \multirow[t]{2}{*}{ This work } & Measurement & $18.6 \pm 2.0$ & $9.9 \pm 1.3$ & $48.3 \pm 5.5$ & $51.8 \pm 2.5$ & $39.7 \pm 5.7$ \\
\hline & Geant4 & 21.1 & 20.4 & 49.6 & 70.9 & 44.1 \\
\hline Breier et al. [21] & CONUS [35] & 14 & 10 & 50 & 76 & 92 \\
\hline \multirow[t]{3}{*}{ Zhang et al. [20] } & Geant4 & 12.31 & 10.32 & 67.15 & 57.26 & 64.63 \\
\hline & ACTIVIA & 30.00 & 20.13 & 77.45 & 138.06 & 66.12 \\
\hline & ACTIVIA $^{1}$ & 14.32 & 8.74 & 32.44 & 56.61 & 26.28 \\
\hline \multirow[t]{2}{*}{ Cebrian et al. [18] } & MENDL+YIELDX ${ }^{2}$ & 32.5 & 22.9 & 88.3 & 159.6 & 97.4 \\
\hline & MENDL+YIELDX 3 & 27.7 & 20.0 & 74.1 & 123.0 & 55.4 \\
\hline \multirow[t]{2}{*}{ Baudis et al. [19] } & Measurement & $13.3_{-2.9}^{+3.0}$ & $9.3_{-1.4}^{+1.2}$ & $44.8_{-8.2}^{+8.6}$ & $68.9_{-5.0}^{+5.4}$ & $29.4_{-5.9}^{+7.1}$ \\
\hline & COSMO & 13.5 & 7.0 & 30.2 & 54.6 & 25.7 \\
\hline Laubenstein et al. [17] & Measurement & $8.85 \pm 0.86$ & $9.5 \pm 1.2$ & $73.8 \pm 16.7$ & $67.9 \pm 3.7$ & $86.2 \pm 7.6$ \\
\hline
\end{tabular}

${ }^{1}$ Calculation relies on cosmic neutron spectra from Gordon [36]

${ }^{2}$ Calculation relies on cosmic neutron spectra from Ziegler [37]

${ }^{3}$ Calculation relies on cosmic neutron spectra from Gordon [36]

$t_{\text {cool }}$ are the time of manufacture, transportation above ground and storage underground, respectively; and $F$ describes the shielding effect for transportation with a steel or lead cover.

Each germanium detector unit has $0.96 \mathrm{~kg}$ of copper and a total of $91.4 \mathrm{~kg}$ of copper well be used for the germanium detector array, as shown in Fig. 3. For simplicity, we assume that the copper components followed the same exposure history as the detectors. The manufacturing time, transportation time and underground storage time are supposed to be three months, a half-month and one year, respectively. After three months of fabrication, the detectors will be transported with shielding, where cosmogenic activations could be reduced by a factor of $10[9,12]$, to the underground laboratory in a half-month. The manufacturing factory is selected to be at sea level, while the altitude of the whole transportation routine is selected to be $1500 \mathrm{~m}$, which is the average altitude of Liangshan Prefecture, where CJPL is located. The cupreous components will be stored in CJPL for preparation work, with the decay of cosmogenic nuclides, the so-called cooling time, lasting about one year.

Importing the measured production rates of the cosmogenic activations, the considered activities in the simulation were obtained using Eq. (5) and the background contributions from cupreous components were estimated with a Geant4based Monte Carlo framework called Simulation and Analysis of Germanium Experiment (SAGE) [38]. In the simulation, these cosmogenic radionuclides were distributed uniformly across all cupreous components, as shown in Fig. 3 . The energy spectra of the different cosmogenic nuclides and the total background contributions were simulated, as shown in Fig. 4.

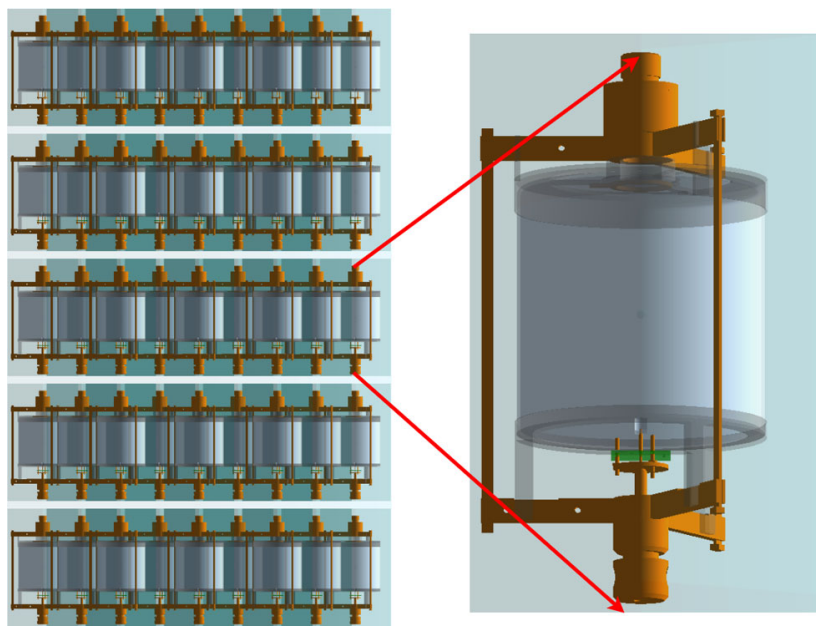

Fig. 3 Sketch of PPCGe detector array (left) and PPCGe detector unit (right) implemented in SAGE, together with its cupreous components, shown in orange. The PPCGe detector array is immersed in liquid nitrogen in the simulation

Under the above assumptions, the simulated background rates in the low-energy range ( $2-4 \mathrm{keV}$ for dark matter search) and around $2039 \mathrm{keV}$ (energy of neutrinoless double beta decay of ${ }^{76} \mathrm{Ge}$ ) are $7.17 \times 10^{-2}$ counts per keV per tonne per year (cpkty) and $1.82 \times 10^{-3}$ cpkty, respectively. The CDEX-100 experiment aims at constructing a germanium detector array with ultra-low background rates, which should be less than 3650 and 0.1 cpkty for the $2-4 \mathrm{keV}$ and around $2039 \mathrm{keV}$ energy regions, respectively. The background contribution from copper can be ignored for dark matter search, while it is $2 \%$ of the total background budget for neutrinoless double beta decay research. The contribution of ${ }^{57} \mathrm{Co}$ dom- 


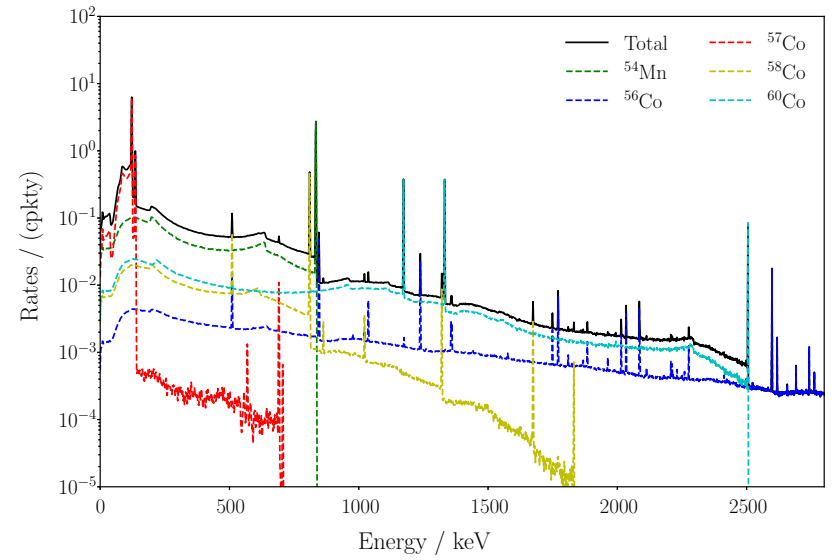

Fig. 4 Simulated cosmogenic background spectra in $0-2.8 \mathrm{MeV}$ region from copper components of germanium detectors for CDEX100 experiment. Energy resolution is determined by the germanium detector used in CDEX-1B [14]

inates the cosmogenic background contributions less than $122 \mathrm{keV}$, and ${ }^{54} \mathrm{Mn}$ is dominant below $830 \mathrm{keV}$, as shown in Fig. $4 .{ }^{60} \mathrm{Co}$ prevails in the higher-energy region among these cosmogenic radionuclides, while only the cosmogenic background exists from ${ }^{56} \mathrm{Co}$ over $2.5 \mathrm{MeV}$. Among these cosmogenic nuclides, only ${ }^{60} \mathrm{Co}$ has a half-life over one year, which is difficult to significantly suppress through underground cooling. A feasible method to mitigate the contribution from ${ }^{60} \mathrm{Co}$ is to reduce the exposure time above ground, or to electroform the copper underground.

\section{Summary}

We investigated the cosmogenic activation in OFHC copper bricks. The specific activities of cosmogenic radionuclides in the exposed copper bricks at the altitude of $2469.4 \mathrm{~m}$ were measured with a low-background germanium gamma-ray spectrometer. The production rates of several long-lived cosmogenic radionuclides were calculated and compared with those of previous studies. The production rates at sea level, in units of nuclei $/ \mathrm{kg} /$ day are $18.6 \pm 2.0$ for ${ }^{54} \mathrm{Mn}, 9.9 \pm 1.3$ for ${ }^{56} \mathrm{Co}, 48.3 \pm 5.5$ for ${ }^{57} \mathrm{Co}, 51.8 \pm 2.5$ for ${ }^{58} \mathrm{Co}$, and $39.7 \pm 5.7$ for ${ }^{60} \mathrm{Co}$, respectively. For comparison, we simulated their cosmogenic activation with Geant4, showing that the agreement between measurement and simulation was generally satisfactory. A general comparison with other published results is presented, showing discrepancies but also good agreement in some cases.

Based on the results of the production rates of cosmogenic radionuclides in copper, the cosmogenic background from copper components of detectors was simulated for the CDEX-100 experiment. The total background rates from cosmogenic nuclides are $7.2 \times 10^{-2}$ cpkty and $1.8 \times 10^{-3}$ cpkty for the $2-4 \mathrm{keV}$ and around $2039 \mathrm{keV}$ energy regions, respectively. The cosmogenic background is dominated by ${ }^{57} \mathrm{Co},{ }^{54} \mathrm{Mn}$ and ${ }^{60} \mathrm{Co}$ after one year of cooling underground. Compared with the total background budget of CDEX-100, the cosmogenic contribution in copper components can be ignored for dark matter search in the region of $2-4 \mathrm{keV}$. Cosmogenic activation in germanium crystals, another crucial background source for CDEX-100, is being studied following the procedure established in CDEX-1B [12].

Acknowledgements This work was supported by the National Key Research and Development Program of China (No. 2017YFA0402201), National Natural Science Foundation of China (Nos. 12175112, 11675088, 11725522, U1865205), and Tsinghua University Initiative Scientific Research Program. Thanks to colleagues of CJPL for their help in copper sample measurements.

Data Availability Statement This manuscript has no associated data or the data will not be deposited. [Authors' comment: The datasets generated during and/or analysed during the current study are available from the corresponding author on reasonable request.]

\section{Declarations}

Conflict of interest The authors have no relevant financial or nonfinancial interests to disclose.

Open Access This article is licensed under a Creative Commons Attribution 4.0 International License, which permits use, sharing, adaptation, distribution and reproduction in any medium or format, as long as you give appropriate credit to the original author(s) and the source, provide a link to the Creative Commons licence, and indicate if changes were made. The images or other third party material in this article are included in the article's Creative Commons licence, unless indicated otherwise in a credit line to the material. If material is not included in the article's Creative Commons licence and your intended use is not permitted by statutory regulation or exceeds the permitted use, you will need to obtain permission directly from the copyright holder. To view a copy of this licence, visit http://creativecomm ons.org/licenses/by/4.0/.

Funded by $\mathrm{SCOAP}^{3}$.

\section{References}

1. G. Heusser, Low-radioactivity background techniques. Annu. Rev. Nucl. Part. S. 45(1), 543-590 (1995)

2. N. Abgrall et al., The MAJORANA DEMONSTRATOR radioassay program. Nucl. Instrum. Methods A 828, 22-37 (2016)

3. D.S. Leonard et al., Trace radioactive impurities in final construction materials for EXO-200. Nucl. Instrum. Methods A 871, 169179 (2017)

4. E. Aprile et al., Material radioassay and selection for the XENON1T dark matter experiment. Eur. Phys. J. C 77(12), 890 (2017)

5. D.S. Akerib et al. The LUX-ZEPLIN (LZ) radioactivity and cleanliness control programs. Eur. Phys. J. C 80(11) (2020)

6. H. Ma et al., In-situ gamma-ray background measurements for next generation CDEX experiment in the China Jinping Underground Laboratory. Astropart. Phys. 128, 102560 (2021) 
7. H. Jiang et al., Limits on Light Weakly Interacting Massive Particles from the First $102.8 \mathrm{~kg} \times$ day Data of the CDEX-10 Experiment. Phys. Rev. Lett. 120(24) (2018)

8. M. Agostini et al., Background-free search for neutrinoless double$\beta$ decay of ${ }^{76}$ Ge with GERDA. Nature 544(7648), 47-52 (2017)

9. N. Abgrall et al., The MAJORANA DEMONSTRATOR Neutrinoless Double-Beta decay experiment. Adv. High Energy Phys. 2014(4), 1-18 (2014)

10. C. Alduino et al., The projected background for the CUORE experiment. Eur. Phys. J. C 77, 543 (2017)

11. D.M. Mei, Z.B. Yin, S.R. Elliott, Cosmogenic production as a background in searching for rare physics processes. Astropart. Phys. 31(6), 417-420 (2009)

12. J.L. Ma et al., Study on cosmogenic activation in germanium detectors for future tonne-scale CDEX experiment. Sci. China Phys. Mech. 62, 08 (2018)

13. Z.Z. Liu et al. Constraints on Spin-Independent Nucleus Scattering with sub-GeV Weakly Interacting Massive Particle Dark Matter from the CDEX-1B Experiment at the China Jinping Underground Laboratory. Phys. Rev. Lett. 123, 161301 (2019)

14. L.T. Yang et al., Search for light weakly-interacting-massiveparticle dark matter by annual modulation analysis with a pointcontact Germanium detector at the China Jinping Underground Laboratory. Phys. Rev. Lett. 123, 221301 (2019)

15. Z. She et al., Direct detection constraints on dark photons with the CDEX-10 experiment at the China Jinping Underground Laboratory. Phys. Rev. Lett. 124, 111301 (2020)

16. Y. Wang et al., Improved limits on solar axions and bosonic dark matter from the CDEX-1B experiment using the profile likelihood ratio method. Phys. Rev. D 101, 052003 (2020)

17. M. Laubenstein, G. Heusser, Cosmogenic radionuclides in metals as indicator for sea level exposure history. Appl. Radiat. Isot. 67(5), 750-754 (2009)

18. S. Cebrián et al., Cosmogenic activation in germanium and copper for rare event searches. Astropart. Phys. 33(5), 316-329 (2010)

19. L. Baudis et al., Cosmogenic activation of xenon and copper. Eur. Phys. J. C 75(10), 1-9 (2015)

20. C. Zhang et al., Cosmogenic activation of materials used in rare event search experiments. Astropart. Phys. 84, 62-69 (2016)

21. R. Breier et al., Monte-Carlo calculation of production rates of cosmogenic radionuclides in a HPGe detector operating in the Modane underground laboratory. Nucl. Instrum. Methods A 978, 164355 (2020)

22. Y.C. Wu et al., Measurement of Cosmic Ray Flux in China JinPing underground Laboratory. Chin. Phys. C 37(8), 45-49 (2013)

23. Z.Y. Guo et al., Muon flux measurement at China Jinping Underground Laboratory. Chin. Phys. C 45, 1 (2021)
24. Z. Zeng et al., The characteristics of a low background germanium gamma ray spectrometer at China JinPing underground laboratory. Appl. Radiat. Isot. 91(11), 165-170 (2014)

25. G.R. Gilmore, Practical Gamma-Ray Spectrometry, 2nd edn. (Wiley, New York, 2008)

26. S. Agostinelli et al., Geant4-A simulation toolkit. Nucl. Instrum. Methods A 506(3), 250-303 (2003)

27. J. Allison et al., Geant 4 developments and applications. IEEE T. Nucl. Sci. 53(1), 270-278 (2006)

28. J. Allison et al., Recent developments in Geant4. Nucl. Instrum. Methods 835(11), 186-225 (2016)

29. P. Goldhagen et al., Measurement of the energy spectrum of cosmicray induced neutrons aboard an ER-2 high-altitude airplane. Nucl. Instrum. Methods A 476(1), 42-51 (2002)

30. N. Lifton, T. Sato, T.J. Dunai, Scaling cosmogenic nuclide production rates using analytical approximations to atmospheric cosmicray fluxes. Earth Planet. Sci. Lett. 386(1), 149-160 (2014)

31. J.F. Ziegler et al., IBM experiments in soft fails in computer electronics (1978-1994). IBM J. Res. Dev. 40(1), 3-18 (1996)

32. S. Cebrian, Cosmogenic activation in double beta decay experiments. Universe 6(10) (2020)

33. J. Apostolakis et al., Geometry and physics of the Geant 4 toolkit for high and medium energy applications. Radiat. Phys. Chem. 78(10), 859-873 (2009)

34. C. Hagmann, D. Lange, D. Wright, Cosmic-ray shower generator (CRY) for Monte Carlo transport codes. IEEE Nucl. Sci. Conf. R. 2, 1143-1146 (2007)

35. J. Masarik, J. Beer, Simulation of particle fluxes and cosmogenic nuclide production in the Earth's atmosphere. J. Geophys. Res. Atmos. 104(D10), 12099-12112 (1999)

36. M.S. Gordon et al., Measurement of the flux and energy spectrum of cosmic-ray induced neutrons on the ground. IEEE Trans. Nucl. Sci. 51(6), 3427-3434 (2004)

37. J.F. Ziegler, Terrestrial cosmic ray intensities. IBM J. Res. Dev. 42(1), 117-140 (1998)

38. Z. She et al., SAGE: a monte carlo simulation framework for experiments with germanium detectors. J. Instrum. 16(09), T09005 (2021) 